\title{
Evaluación de la Influencia en la Calidad de Alimento para Pollo de Engorde Utilizando Acondicionamiento con Aplicación de Vapor en Varios Puntos
}

\author{
Evaluation of the Influence on Quality of Broller Feed Using \\ Steam Conditioning With Application at Various Points
}

\section{Covaleda Triana Ninfa}

Consultor de calidad. Barranquilla Colombia, ing.ncovaleda@hotmail.com

Recibido: 4/09/2011 • Aprobado: 5/12/2011

\section{RESUMEN}

El presente artículo muestra un desarrollo experimental para el mejoramiento del acondicionamiento de alimentos balanceados para pollo de engorde, trabajo realizado con el objetivo de mejorar y evaluar el comportamiento del proceso de vapor con varias entradas, en una empresa que produce 4.000 ton/mes. La adaptación de la nueva tecnología se realizó con el fin de aumentar la eficiencia del proceso de elaboración de alimento balanceado, mejorando la calidad del mismo, con un nivel bajo de inversión.

Durante el desarrollo experimental, se muestran los beneficios generados en términos productivos y de calidad, los cuales fueron satisfactorios para el modelo.

Palabras clave: acondicionamiento, calidad, eficiencia, mejoramiento, vapor

\section{ABSTRACT}

His paper presents experimental development to improve the conditioning of feed for broiler work in order to improve and evaluate the behavior of the conditioning process of balanced feed for Broiler with steam distribution with multiple entries in a company producing 4000 ton / month, the adaptation of new technology was performed in order to increase the efficiency of the process of making balanced food improving its quality with a low investment. During the experimental development profits generated in terms of production and quality are shown, which were satisfactory for the experimental model.

Keywords: conditioning, efficiency, improvement, quality, vapor 


\section{INTRODUCCIÓN}

El presente artículo muestra una adaptación tecnológica al proceso de acondicionamiento de alimentos balanceados para pollo de engorde, que permite la mejora del proceso sin mayor inversión, en cuanto a calidad y eficiencia del equipo experimental vs el tradicional.

Los alimentos para animales son mezclas de ingredientes elaborados en forma tal que respondan a requerimientos nutricionales para cada especie, edad, estado productivo y tipo de explotación [1].

En Colombia, la industria de alimentos balanceados llega a una producción anual de alrededor de $5.800 .000 \mathrm{tm}$, de la cual, aproximadamente, el $70 \%$ corresponde a pollo de engorde [2]. Teniendo en cuenta lo anterior y la alta competitividad de la industria en Colombia, se realizó un trabajo experimental del proceso de acondicionamiento de alimentos balanceados, el cual consiste en aumentar la digestibilidad de almidones, mejorar las características reológicas de la masa y la calidad higiénico sanitario de los productos y acrecentar el rendimiento del proceso de elaboración en ton/h. Este proceso es uno de los que genera mayor costo en la elaboración del alimento por su alto consumo de energía; por tal motivo, se decide evaluar su comportamiento al inyectar vapor en varios puntos; es decir, distribuir el vapor de una mejor manera dentro del acondicionador, con el fin de mejorar los parámetros anteriormente mencionados, especialmente el de la calidad y el de la productividad.

Para la ejecución del experimento se realizó seguimiento, por un mes, a los parámetros de producción del nuevo método, y se compararon los resultados de eficiencia, \%, durabilidad y \% de finos vs el método tradicional.

Este análisis se evaluó estadísticamente, aplicando el de Anova de una sola vía y se mostró que existen diferencias estadísticas significativas entre los dos métodos y, así mismo, que la nueva metodología es muy eficiente para el mejoramiento del proceso de acondicionamiento, ya que se observó una mejora significativa en la presentación del alimento balanceado y un aumento en las ton/h, gracias a la estabilidad del equipo, lo que favorece la rentabilidad de la planta al producir muchas más toneladas de alimento con la misma capacidad instalada.

\section{Marco Conceptual}

El procesamiento de alimentos balanceados ha tenido una gran evolución en cuanto a las técnicas de producción y, específicamente, en relación con el proceso de acondicionamiento, para lo cual se han realizado gran cantidad de trabajos y propuestas que redundan en el mejoramiento del mismo, con el fin de obtener los mejores resultados. Para tal fin, la primera tecnología utilizada fue la del peleteado con acondicionamiento simple, proceso en el que ocurre una reducción microbiológica, en menor escala, pasando, posteriormente, por los expanders y los acondicionadores dobles y triples. Con todos estos avances se pretende mejorar las condiciones de digestibilidad del alimento, así como la calidad del pellet.

\section{A. Descripción de proceso de elaboración de balanceados.}

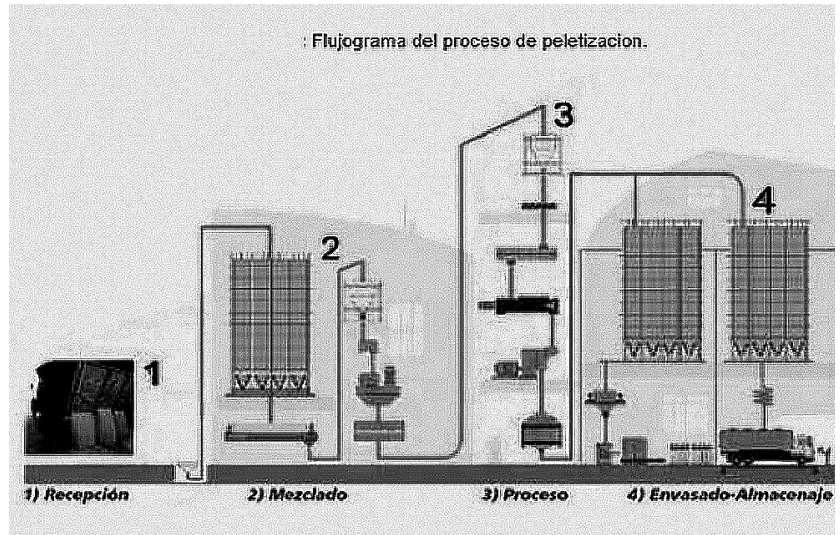

Fig. 1 Diagrama de flujo elaboración de alimento balanceado [3]. 
A continuación se describe brevemente el proceso de elaboración de balanceados:

1) Recepción de materias primas: en esta etapa del proceso se reciben las materias primas utilizadas en la elaboración de alimentos balanceados, entre otras, maíz, torta de soya, subproductos de origen animal, gluten, aceites y aminoácidos. Durante este proceso se realizan análisis físicos, químicos y microbiológicos.

2) Almacenamiento: en esta etapa, las materias primas se almacenan en silos o bodegas, hasta el momento en que se utilicen en el proceso de elaboración. Durante el almacenamiento es importante realizar control de materias primas, en cuanto a humedad, temperatura y grado de infestación.

3) Molienda: proceso mediante el cual se pretende la reducción de tamaño de las partículas, especialmente del maíz, con el fin de que sea más digestible para el animal. Este proceso se realiza, generalmente, con molinos de martillos y es de gran importancia dentro de la planta, ya que dependiendo de la granulometría, los resultados zootécnicos se verán altamente beneficiados.

4) Dosificación: consiste en adicionar las materias primas necesarias según la formulación. Este procedimiento se realiza por medio de unas tolvas dosificadoras, las cuales cuentan con unos sensores, o también de forma manual dependiendo de la planta.

5) Mezclado: el mezclado es uno de los procesos más importantes en la elaboración de alimentos balanceados ya que su objetivo principal es crear una mezcla homogénea que logre cumplir todos los requerimientos nutricionales de la especie en la fase de desarrollo específico para la cual se creó la fórmula [4].

6) Acondicionamiento: el acondicionamiento de alimentos balanceados es un proceso mediante el cual se aplica vapor (humedad y calor) y/o presión a la mezcla de alimento balanceado por un periodo específico de tiempo [5]. Es importante aplicar vapor "seco", pues la humedad del vapor que ingresa y se mezcla con el producto debe ser mínima para tener mayor eficiencia en la transferencia de calor, mejorando así el proceso de acondicionamiento [6].

En esta etapa solo se aplica calor sensible, que es aquel que recibe un cuerpo y hace que aumente su temperatura sin afectar su estructura molecular y, por lo tanto, su estado, en general. Se ha observado experimentalmente que la cantidad de calor necesaria para calentar o enfriar un cuerpo es directamente proporcional a la masa del cuerpo y al número de grados en que cambia su temperatura. La constante de proporcionalidad recibe el nombre de calor específico [7].

Durante la aplicación de vapor al producto mezclado, se quiere obtener una masa con características reológicas aptas para el proceso de peletizado (que los pellets sean consistentes), mejorar la inocuidad de la masa y favorecer el desdoblamiento de almidones; es decir, se realiza una cocción del producto para mejorar sus características nutricionales que favorezcan los resultados de campo ya, que con este proceso y posterior peletizado, se garantiza que a cada animal le lleguen raciones uniformes.

Los rangos de entrada de los alimentos al acondicionador se encuentran entre un $11 \%$ y $12 \%$; a la salida del acondicionador se consiguen humedades entre 15 y $17 \%$.

Finalizado el acondicionamiento, el producto está listo para ser convertido en pellet.

7) Peletizado: el peletizado es una operación de moldeo termoplástico en el que partículas de una ración, finamente divididas, se integran en un pellet compacto y de fácil manejo, el cual incluye condiciones específicas de humedad, temperatura y presión. Aspectos tales como dureza y grado de compactación de los pellets, lo determinan el diámetro de las perforaciones de la matriz y 
el largo o recorrido que debe hacer el alimento para recorrer estas perforaciones. Esta relación es variable de 10:1 hasta 16:1; cuanto mayor es la relación, es de esperarse que la dureza del pellet sea mayor [8].

8) Enfriamiento: este proceso tiene como finalidad bajar la temperatura y la humedad de los pellets haciendo pasar una corriente de aire a través de ellos; se recomienda secar los productos a $12,5 \%$ de humedad y a una temperatura no mayor de $5^{\circ} \mathrm{C}$, por encima de la temperatura ambiente.

9) Empaque: en esta etapa del proceso se empacan cada una de las referencias de alimentos producidas en bultos de $40 \mathrm{~kg}$, los cuales deben estar marcados y etiquetados correctamente para la trazabilidad del producto; se realiza, también, la verificación de las características físicas del producto terminado: análisis de durabilidad y \% de finos.

\section{B. Descripción de las variables evaluadas durante el experimento}

1) Durabilidad: capacidad relativa de los peIlets para resistir la rotura durante el transporte, almacenamiento y descargue [9]; su valor normal está dado para alimento de pollo de engorde en un rango de 91 - $94 \%$. Es un valor comercial muy importante del alimento paleteado, ya que se requiere que este llegue entero desde la planta al galpón.

2) Porcentaje de finos: los finos corresponden a la cantidad de material que queda sin ser peletizada y que tienen un tamaño de partícula inferior a 600 micras. Entre mayor cantidad de finos haya en el alimento, mayor será la cantidad de producto que se desperdicia en los comederos de los galpones lo que resulta una pérdida económica para los productores de pollo.

\section{Metodología}

El experimento se desarrolló en una planta de alimentos balanceados, que produce $4000 \mathrm{tn} / \mathrm{h}$ de alimento para pollo.

Después de hacer un análisis sobre la productividad de los equipos y la calidad del alimento balanceado y teniendo en cuenta la necesidad del industrial de aumentar su productividad, se procede a proponer el ensayo de la adaptación tecnológica en la planta, que consiste en la distribución del vapor en varios puntos hasta la mitad del cuerpo del acondicionador, (originalmente los acondicionadores solo traen una sola entrada de vapor en la parte inicial de su estructura), permitiendo que la mezcla entre en contacto con el vapor por un corto periodo de tiempo; se busca que este contacto sea mayor para mejorar las características del producto.

En la planta existen dos peletizadoras, una de las cuales se escogen por baja productividad y se le realiza la adaptación tecnológica; la otra queda en las condiciones normales de operación con el objetivo de realizar el comparativo entre la nueva metodología y la tradicional.

\section{A. Adaptación de acondicionador con siete puntos de inyección de vapor}

Se instalaron 7 puntos de inyección de vapor cada $10 \mathrm{~cm}$, ya que el acondicionador tiene una longitud de 2 metros. Lo anterior, teniendo en cuenta, la necesidad de instalar la distribución de vapor en la primera mitad del acondicionador, puesto que si se hacía en todo el cuerpo, el alto porcentaje de humedad que se ganaría, podría causar atascamiento en el proceso, perjudicando, así, el funcionamiento normal del equipo.

De tal manera, se hicieron las siguientes mediciones para lograr esta condición: $70 \mathrm{~cm}$ da la suma de la separación entre los puntos de inyección de vapor, más el diámetro de los puntos, cada uno de $5.08 \mathrm{~cm}$; siendo 7 puntos da en total 
$35.56 \mathrm{~cm}$ ocupados por la tubería, y si le sumamos la separación entre cada punto, da un total de $105,56 \mathrm{~cm}$, la medida para alcanzar la mitad del cuerpo del acondicionador, lo que da como resultado, en esta primera sección, mayor contacto del producto con el vapor.

Los acondicionadores son de la misma marca y presentan condiciones de operaciones similares, así como la misma capacidad, $10 \mathrm{tn} / \mathrm{h}$; el acondicionador comparativo continuó trabajando en las condiciones normales de operación, con la finalidad de comparar los dos métodos.

Las siete entradas de vapor en el acondicionador se instalaron con los siguientes materiales:

- 7 tubos de acero al carbón de 2 pulgadas.

- 7 válvulas de $3 / 4$.

- 1 tubería de 1 metro de 3/4 de pulgadas.

- Fibra de vidrio.

- Cubierta de aluminio.

La ubicación y distribución de los puntos de vapor se observan en la Fig. 2:

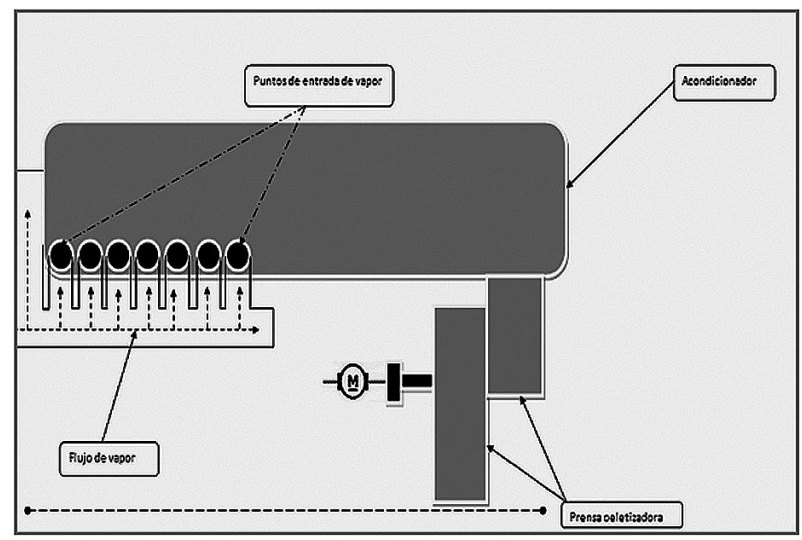

Fuente: El autor.

Fig. 2 Instalación de puntos de vapor en el acondicionador.

\section{B. Evaluación de la influencia en la calidad}

La evaluación de este proceso se realizó durante un mes. Se revisó y registró cada uno de los parámetros medidos en los acondicionadores. Las mediciones se realizaron 6 veces al día, para un total de 180 mediciones, por cada uno de los acondicionadores, para el caso de la durabilidad $\mathrm{y}$ de los finos.

Se evaluaron los siguientes parámetros:

Calidad física del producto: \%durabilidad, \% finos [10]. Los parámetros microbiológicos no se tuvieron en cuenta para este experimento debido al alto costo de los análisis.

\section{Evaluación del impacto a la eficiencia del proceso}

El impacto en la eficiencia se evaluó teniendo en cuenta la producción de mayor ton $/ \mathrm{h}$, con la misma capacidad instalada; para esto se valoraron las toneladas producidas por cada uno de los equipos y se compararon para evaluar. En total se realizaron 3 mediciones al día, para un total de 90 , las cuales se valoraron mediante el análisis Anova de un factor.

\section{Comportamiento de las variables de operación.}

Se graficó el comportamiento de las variables de humedad, temperatura y presión de cada uno de los equipos, con el fin de observar con cuál de las dos metodologías se obtenía mejores condiciones de operación. Para esto, se graficaron los promedios; de cada 18 observaciones en total se graficaron 10 datos promedios; además, para cada variable, se realizó análisis de c.v.

\section{E. Análisis estadístico}

Para el desarrollo experimental se plantearon las siguientes hipótesis:

Hipótesis nula:

HO: La inyección de vapor en diferentes puntos no genera mayor eficiencia ni calidad en el proceso de acondicionamiento.

Hipótesis alternativa:

H1: La inyección de vapor en diferentes puntos genera mayor eficiencia y calidad en el proceso de acondicionamiento. 


\section{F. Condiciones del vapor por utilizar}

La cantidad de vapor por utilizar en el proceso se calculó como se muestra a continuación:

\section{Cálculo de la cantidad de vapor requerido}

Ecuación (1) = $470 \mathrm{~kg} / \mathrm{h}$ de vapor

En general, para las condiciones del proceso se requiere vapor saturado a 100 psi y $165^{\circ} \mathrm{C}$.

\section{Resultados}

A continuación se relacionan los resultados para cada una de las variables analizadas durante el experimento.

TABLA I

Análisis Estadístico Anova de un Factor para el \% Durabilidad

\begin{tabular}{lcccc}
\multicolumn{5}{c}{ ANÁLISIS DE VARIANZA DE UN FACTOR } \\
\multicolumn{4}{c}{ RESUMEN } \\
Grupos & Cuenta & Suma & Promedio & Varianza \\
Experimental & 180 & 16746,5 & 93,03583333 & 2,27664679 \\
Tradicional & 180 & 15873,9 & 88,18846667 & 3,2147356 \\
\hline
\end{tabular}

\begin{tabular}{lcccccc}
\multicolumn{1}{c}{$\begin{array}{c}\text { Origen de las } \\
\text { variaciones }\end{array}$} & $\begin{array}{c}\text { Suma de } \\
\text { cuadrados }\end{array}$ & $\begin{array}{c}\text { Grados de } \\
\text { libertad }\end{array}$ & $\begin{array}{c}\text { Promedio de los } \\
\text { cuadrados }\end{array}$ & F & Probabilidad & $\begin{array}{c}\text { Valor crítico } \\
\text { para F }\end{array}$ \\
\hline $\begin{array}{l}\text { Entre grupos } \\
\text { Dentro de los }\end{array}$ & 2114,72672 & 1 & 2114,726724 & 770,198312 & $2,99 \mathrm{E}-91$ & 3,86756452 \\
$\begin{array}{l}\text { grupos } \\
\text { Total }\end{array}$ & 982,957448 & 358 & 2,745691195 & & & \\
\hline
\end{tabular}

El resultado para la durabilidad indica que se $\quad=0,05$, ya que el valor de $F>F$ critica, lo que acepta $\mathrm{H} 1$ :

La inyección de vapor en diferentes puntos genera mayor calidad en el proceso de acondicionamiento en un nivel de significancia de $\mathrm{F}$ indica que existe diferencia estadística para el resultado de la durabilidad de los dos equipos, siendo mejor el arrojado por el acondicionador experimental.

TABLA II

Análisis de Varianza Anova de un Factor para el Porcentaje de Finos

\begin{tabular}{lcccc}
\multicolumn{5}{c}{ ANÁLISIS DE VARIANZA DE UN FACTOR } \\
\multicolumn{5}{c}{ RESUMEN } \\
\multicolumn{1}{c}{ Grupos } & Cuenta & Suma & Promedio & Varianza \\
Experimental & 180 & 16746,5 & 93,03583333 & 2,27664679 \\
Tradicional & 180 & 15873,9 & 88,18846667 & 3,2147356 \\
\hline
\end{tabular}

\begin{tabular}{|c|c|c|c|c|}
\hline \multicolumn{5}{|c|}{ ANÁLISIS DE VARIANZA DE UN FACTOR } \\
\hline \multicolumn{5}{|c|}{ RESUMEN } \\
\hline Grupos & Cuenta & Suma & Promedio & Varianza \\
\hline Experimental & 180 & 1421,62 & 7,89788889 & 4,01141675 \\
\hline Tradicional & 180 & 2462,7 & 13,6816667 & 5,15591899 \\
\hline
\end{tabular}


ANÁLISIS DE VARIANZA

\begin{tabular}{lcccccc}
$\begin{array}{l}\text { Origen de las } \\
\text { variaciones }\end{array}$ & $\begin{array}{c}\text { Suma de } \\
\text { cuadrados }\end{array}$ & $\begin{array}{c}\text { Grados de } \\
\text { libertad }\end{array}$ & $\begin{array}{c}\text { Promedio de los } \\
\text { cuadrados }\end{array}$ & F & Probabilidad & $\begin{array}{c}\text { Valor crítico } \\
\text { para F }\end{array}$ \\
\hline Entre grupos & 3010,68768 & 1 & 3010,68768 & 656,829371 & $5,2387 \mathrm{E}-83$ & 3,86756452 \\
$\begin{array}{l}\text { Dentro de los } \\
\text { grupos }\end{array}$ & 1640,9531 & 358 & 4,58366787 & & & \\
Total & 4651,64078 & 359 & & & & \\
\hline
\end{tabular}

El análisis de varianza Anova para el porcentaje de finos indica que se acepta $\mathrm{H} 1$ :

La inyección de vapor en diferentes puntos genera mayor calidad en el proceso de acondicio- namiento en un nivel de significancia de $\mathrm{F}=0,05$, permitiendo una mayor compactación de los peIlet; por ende, las partículas denominadas finos o materia no peletizado es menor.

TABLA III

AnÁlisis Anova de un Factor para la Eficiencia

\begin{tabular}{lcccc}
\multicolumn{5}{c}{ RESUMEN } \\
\multicolumn{1}{c}{ Grupos } & Cuenta & Suma & Promedio & Varianza \\
Experimental & 91 & 778,1 & 8,55054945 & 0,05519414 \\
Tradicional & 91 & 674,9 & 7,41648352 & 0,0649475 \\
\hline
\end{tabular}

\begin{tabular}{|c|c|c|c|c|c|c|}
\hline \multicolumn{7}{|c|}{ ANÁLISIS DE VARIANZA } \\
\hline $\begin{array}{l}\text { Origen de las } \\
\text { variaciones }\end{array}$ & $\begin{array}{l}\text { Suma de } \\
\text { cuadrados }\end{array}$ & $\begin{array}{l}\text { Grados de } \\
\text { libertad }\end{array}$ & $\begin{array}{l}\text { Promedio de los } \\
\text { cuadrados }\end{array}$ & $\mathbf{F}$ & Probabilidad & $\begin{array}{c}\text { Valor crítico } \\
\text { para } F\end{array}$ \\
\hline Entre grupos & 58,5178022 & 1 & 58,5178022 & 974,146917 & $1,52 \mathrm{E}-74$ & 3,89363972 \\
\hline $\begin{array}{l}\text { Dentro de los } \\
\text { grupos }\end{array}$ & 10,8127473 & 180 & 0,06007082 & & & \\
\hline Total & 69,3305495 & 181 & & & & \\
\hline
\end{tabular}

Para el caso de la eficiencia del equipo se acepta $\mathrm{H} 1$ :

La inyección de vapor en diferentes puntos genera mayor eficiencia en el proceso de acondicionamiento, ya que $F>F$ critica en un nivel de significancia de $F=0,05$.

\section{A. Resultados para el análisis del comporta- miento de las variables}

Para el caso de la humedad, se observa que el equipo con varias entradas de vapor presenta un mayor contenido de humedad, lo que es favorable, ya que según las técnicas de elaboración de balanceados, la humedad en el acondicionador debe estar entre 15 y 17\% [6]; este parámetro se está cumpliendo para el acondicionador experimental.

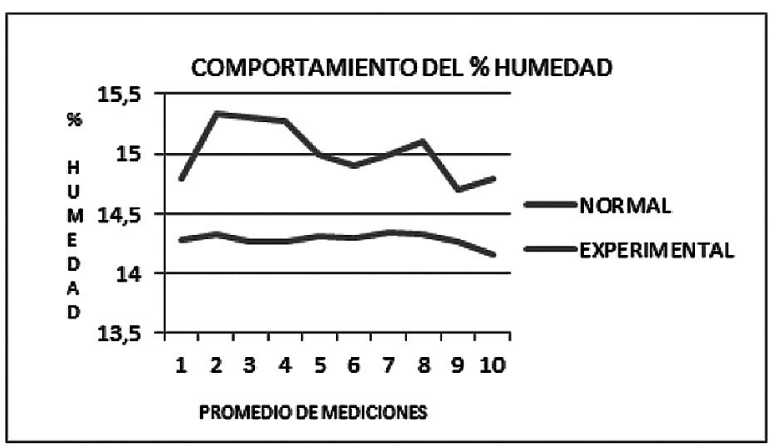

Fuente: El autor

Fig.3 Comportamiento del \% de Humedad. 


\section{B. Análisis del comportamiento de la temperatura}

Para el caso de la temperatura se observa que se obtuvo una mayor temperatura en el acondicionador experimental, lo que indica que el vapor pudo ceder más calor a la masa por la distribución del vapor y mayor contacto de la masa con el mismo; esto influyó en la calidad del producto terminado, ya que se logró un mayor desdoblamiento de almidones, lo que confirió mejores características a la masa.

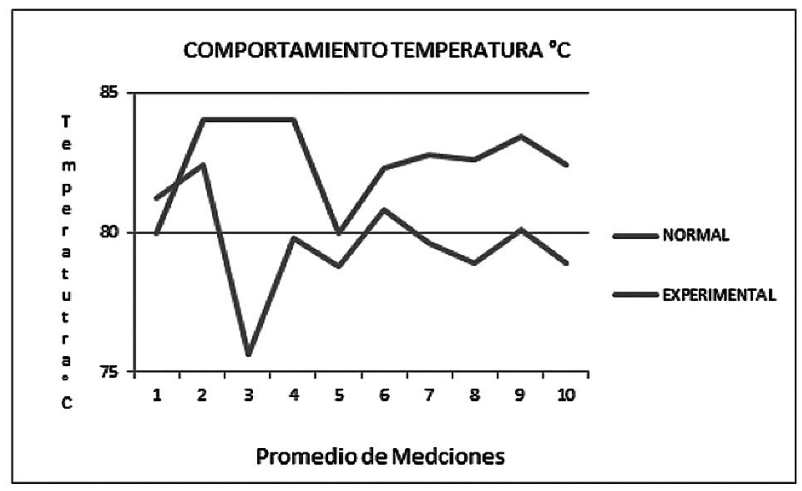

Fuente: El autor

Fig.4 Comportamiento Temperatura ${ }^{\circ} \mathrm{C}$.

\section{Análisis del comportamiento de la presión}

De acuerdo con la Tabla 6, el comportamiento de la presión se mantuvo más estable para el acondicionador experimental, lo que generó menos atascamientos en este equipo.

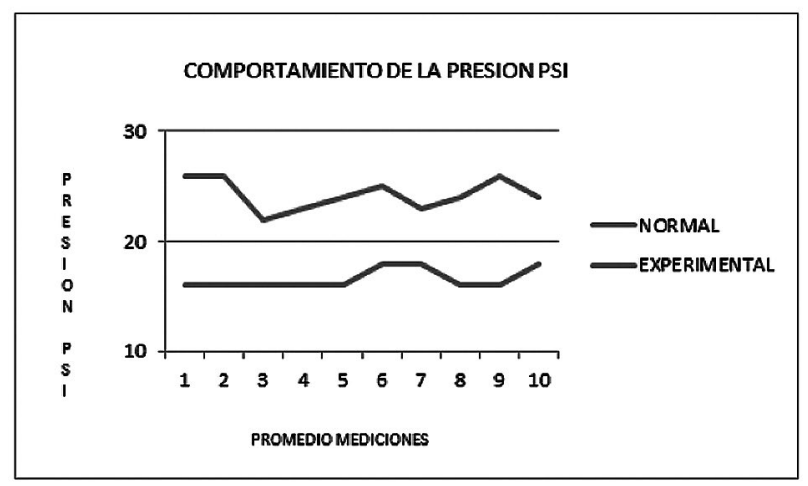

Fuente: el autor.

Fig.5 Comportamiento de la Presión en PSI

\section{Análisis estadístico del comportamiento de las variables.}

En las Tablas 4 y 5 se relacionan los resultados de los análisis estadísticos de los dos acondicionadores:

TABLA IV

Análisis Estadístico para el Acondicionador Tradicional

\begin{tabular}{|c|c|c|c|}
\hline PROMEDIOS & \% H acona & T ${ }^{\circ} \mathrm{C}$ & $\begin{array}{c}\text { PRESION } \\
\text { PSI }\end{array}$ \\
\hline 1 & 14,28 & 81,2 & 26 \\
\hline 2 & 14,32 & 82,4 & 26 \\
\hline 3 & 14,27 & 75,6 & 22 \\
\hline 4 & 14,26 & 79,8 & 23 \\
\hline 5 & 14,31 & 78,8 & 24 \\
\hline 6 & 14,29 & 80,8 & 25 \\
\hline 7 & 14,35 & 79,6 & 23 \\
\hline 8 & 14,32 & 78,9 & 24 \\
\hline 9 & 14,27 & 90,1 & 26 \\
\hline 0 & 14,16 & 78,9 & 24 \\
\hline PROMEDIO & 14,283 & 79,61 & 24,3 \\
\hline DESVIACION & 0,051650535 & 1,81808813 & 1,41813649 \\
\hline C.V. & 0,361622454 & 2,28374341 & 5,83695264 \\
\hline & & & \\
\hline
\end{tabular}

Fuente: El autor

El análisis estadístico arroja que para el acondicionador tradicional, en promedio, el porcentaje de humedad estuvo bajo el $14,23 \%$, por lo que no se cumplió con los parámetros establecidos; aunque el coeficiente de variación para esta variable muestra un comportamiento estable en el producto, para el caso de la temperatura, también estuvo por debajo de la requerida: $79.61 \mathrm{C}$. Para la presión se requirió una mayor cantidad de esta, con el fin de tratar de alcanzar los parámetros requeridos; sin embargo, no fue suficiente para lograr la temperatura adecuada y el coeficiente de variación para esta variable fue mayor; es decir, se presentó gran variación en la presión de operación. 
TABLA V

Análisis Estadístico para el Acondicionador Experimental

\begin{tabular}{|c|c|c|c|}
\hline PROMEDIOS & \% Hacona & T'C & $\begin{array}{c}\text { PRESWN } \\
\text { PISI }\end{array}$ \\
\hline 1 & 14,8 & 80 & 16 \\
\hline 2 & 15,33 & 84 & 16 \\
\hline 3 & 15,3 & 84 & 16 \\
\hline 4 & 15,28 & 84 & 16 \\
\hline 5 & 15 & 80 & 16 \\
\hline 6 & 14,9 & 82,3 & 18 \\
\hline 7 & 15 & 82,8 & 16 \\
\hline 8 & 15,1 & 82,6 & 16 \\
\hline 9 & 14,7 & 83,4 & 16 \\
\hline 10 & 14,8 & 82,4 & 18 \\
\hline PROMEDIO & 15,021 & $\mathrm{B2}, 55$ & 16,4 \\
\hline DESVIACION & 0,226756733 & 1,49759065 & 0,84327404 \\
\hline C.V. & 1,509797836 & 1,81416191 & 5,14191489 \\
\hline
\end{tabular}

Fuente: El Autor

Para el caso del experimental, en promedio, la temperatura y la humedad alcanzaron los requerimientos esperados; se logró trabajar a una presión más baja y se obtuvo una variación baja en casi todos los parámetros. Con este análisis se muestra que se presentaron mejores condiciones para este acondicionador; es decir, las variables del proceso muestran una mayor favorabilidad para el experimental, ajustándose a los parámetros requeridos de humedad y temperatura.

\section{Discusión}

Los resultados arrojados por la estadística muestran para las tres evaluaciones realizadas que la hipótesis nula se acepta; es decir, que en los tres casos el acondicionador con las 7 entradas de vapor muestra una mejor condición para la elaboración del producto, permitiendo que este tenga mayor tiempo de contacto con el vapor, favoreciendo, de esta manera, la calidad de los pellet y la eficiencia del equipo.

A continuación se grafican las variables medidas en el experimento y se comparan con el método tradicional:

\section{A. Comportamiento de la durabilidad}

En la Fig. 6 se observa que el valor de la durabilidad de los pellet en todos los casos fue mejor para el acondicionador con varias entradas de vapor, debido a la mejor transferencia de calor del vapor a la masa y a la mejora en la textura del producto. Se pudieron observar unas diferencias en la temperatura de la masa acondicionada de hasta $2{ }^{\circ} \mathrm{C}$, y humedad de 0,3-0,6\%; estos datos no se analizaron estadísticamente, pero son un indicador de la mejora en la transferencia de calor ya que la masa adquirió mayor temperatura y mayor humedad con la distribución de vapor.

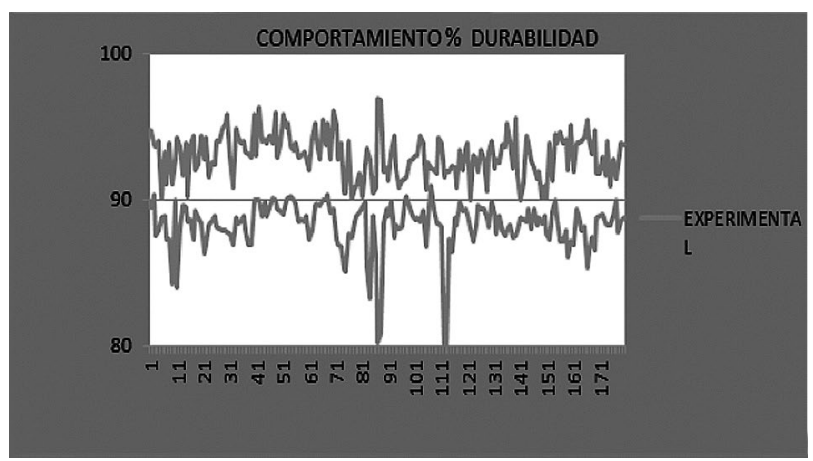

Fuente: El autor

Fig. 6 Comportamiento del \% de durabilidad.

\section{B. Comportamiento del \% de finos}

El comportamiento del \% de finos indica una menor cantidad de partículas sin peletizar para el acondicionador con las entradas de vapor, lo que indica una mayor eficiencia en la prensa peletizadora, ya que se obtuvo una masa de mejor calidad para el proceso de peletizado lo que indica que es mejor trabajar con varias entradas de vapor.

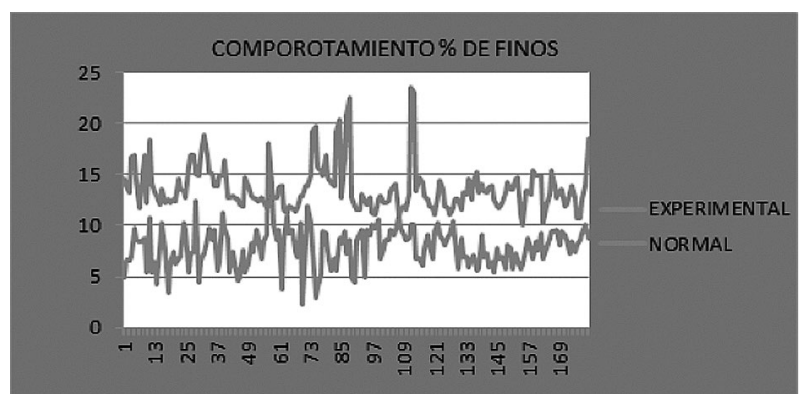

Fuente: El autor

Fig. 7 Comportamiento del \% finos 8. 


\section{Comportamiento en la eficiencia del equipo}

El comportamiento de la eficiencia del equipo arrojó como resultado que, en promedio, hay una diferencia de 1,14 ton/h, a favor de la peletizadora con varias entradas de vapor, lo que es sumamente importante, porque impacta directamente la rentabilidad de la planta. Si la planta trabaja 12 horas al día 13,68 ton más, se produciría en turno de trabajo casi la producción de un día, la cual sería de aproximadamente 15 ton, si las dos peletizadoras trabajan al mismo ritmo. Esto ayuda a reducir el tiempo por horas trabajadas en el mes y a disminuir los costos de consumo de energía y gas.

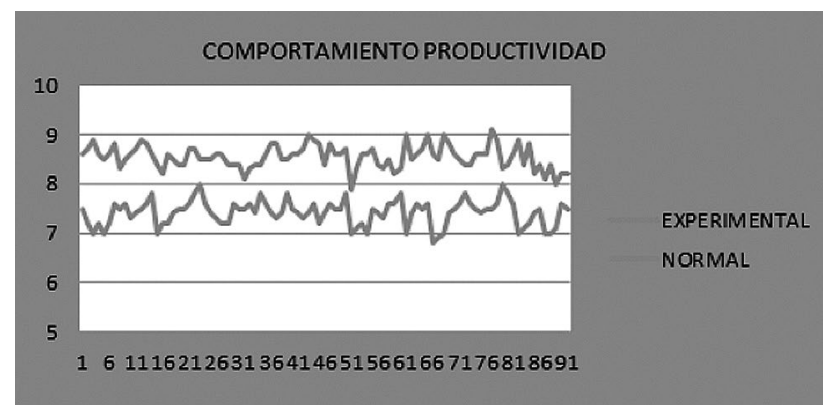

Fuente: El autor

Fig. 8 Comportamiento de la productividad.

\section{Tiempo de retención}

Otro de los parámetros importantes y no evaluados a profundidad en este trabajo, es el tiempo de retención en el acondicionador, el cual es necesario para generar un buen desdoblamiento de almidones y obtener buenos resultados de durabilidad. Para este trabajo, el proceso se realizó con un tiempo de acondicionamiento de aproximadamente 40 seg. El tiempo de retención óptimo para cualquier acondicionador es el tiempo que se requiere para que la humedad y el calor lleguen a cada partícula del pienso; entre mayor sea el tiempo de retención mayor será el grado de gelatinización de almidones y así mayor será la durabilidad de los pellets [11]. En la Fig. 3, se establecen las relaciones entre el tiempo de acondicionamiento y el grado de gelatinización de los almidones:
TABLA VI

DigestibilidAd de Almidones vs. Tiempo de RETEnción [12].

\begin{tabular}{|l|l|l|}
\hline Tipo de equipo & $\begin{array}{l}\text { Tiempo de } \\
\text { retención } \\
\text { (seg) }\end{array}$ & $\begin{array}{l}\text { Porcentaje de } \\
\text { gelatinización }\end{array}$ \\
\hline $\begin{array}{l}\text { Acondicionador } \\
\text { simple }\end{array}$ & 15 a 20 & $15-20$ \\
\hline $\begin{array}{l}\text { Acondicionador } \\
\text { Doble }\end{array}$ & 40 a 45 & $20-25$ \\
\hline $\begin{array}{l}\text { Acondicionador } \\
\text { doble }\end{array}$ & 120 a 180 & $40-50$ \\
\hline
\end{tabular}

\section{Grado de peletizacion de ingredientes}

Los diferentes ingredientes utilizados en la formulación tienen características diversas, en cuanto a tamaño, forma, densidad, peso y grado de peletización; de esta manera, para facilitar el desdoblamiento de los almidones en el caso de la dieta utilizada para el ensayo, se utilizaron, principalmente, el maíz y la torta de soya, materias primas que tienen, según la clasificación de la asociación de fabricantes americanos de piensos, un grado medio de peletización, lo que favorece el desarrollo normal del proceso y la calidad final de los pellets.

Al utilizar materias primas con bajo grado de peletización, muy posiblemente la eficiencia del proceso se vea reducida, ya que estas no resisten, por lo general, las altas temperaturas del proceso, o no son miscibles con el vapor, lo que puede generar desmoronamiento de pellets. Es importante aplicar el proceso a este tipo de materiales y evaluar su eficiencia y calidad, con el propósito de observar si el sistema genera algún tipo de beneficio o, si por el contrario, es necesario utilizar otro tipo de tecnología.

Es indispensable el conocimiento de las materias primas en cuanto a sus características físicas y grado de peletización a la hora de realizar el proceso de peletización, pues es un buen indicativo del comportamiento del producto terminado. 


\section{Conclusiones}

La aplicación de vapor en varios puntos es una alternativa para el mejoramiento de la calidad de los pellet ya que optimiza el \% de durabilidad del producto terminado y disminuye el \% de los finos, así como la presentación de los productos terminados.

La aplicación de vapor en varios puntos mejoró la eficiencia del equipo en 1,14 ton/h, lo que representa un aumento en la producción, con la misma capacidad instalada, y favorece la rentabilidad de la planta; así mismo se pueden reducir las horas trabajadas en esta.

La instalación de los puntos de vapor en el equipo fue favorable, pues no generó inconvenientes en su funcionamiento, ya que no se presentaron atascamientos ni demoras en la producción, por la utilización de los puntos de vapor.

La instalación de vapor en varios puntos mejoró las condiciones de estabilidad de la máquina en Amperaje; observó un comportamiento más estable debido a que la carga de vapor se distribuyó a lo largo del acondicionador y evitó atascamiento del mismo.

Con el experimento realizado se demuestra que es viable instalar los puntos de vapor en el otro acondicionador, para mejorar las características de calidad y eficiencia de la otra máquina.

Es importante mantener y evaluar las condiciones del vapor para el proceso de acondicionamiento, con el fin de lograr una buena transferencia de calor y gelatinización de almidones.

El tiempo de retención en el acondicionador es una variable necesaria para lograr los objetivos en el proceso de acondicionamiento, por cuanto favorece la gelatinización de almidones, la hi- gienización del producto y la textura de la masa acondicionada; el tiempo recomendado, según algunos expertos, es de más de $40 \mathrm{seg}$. De todas maneras, es importante que cada planta y cada proceso evalúe en qué tiempo puede conseguir las condiciones óptimas para sus productos.

Teniendo en cuenta las materias primas utilizadas en la dieta se puede prever el comportamiento del proceso y la influencia que estas puede tener dentro del mismo, en relación con la eficiencia y la calidad.

La industria de alimentos balanceados es una industria cambiante, en continuo desarrollo y con una alta competitividad, lo que genera que la tecnología y los empresarios estén en la búsqueda continua de nuevas opciones de mejora en términos de calidad y eficiencia, con la finalidad de ser competitivos en el mercado.

Las nuevas tecnologías aplicadas al proceso de acondicionamiento de alimentos balanceados están encaminadas a reducir costos de operación y lograr mejores características en los alimentos; sin embargo, se requiere de grandes inversiones iniciales, motivo por el cual, la mayoría de empresarios no pueden acceder a estas y deben buscar otras alternativas que les garantice o les ayude a mantener su permanencia en el mercado.

Colombia es un país con gran potencial en la región, teniendo en cuenta que maneja procesos estandarizados y tecnificados, lo que le permite ser un referente en el sector y favorecer la inversión y crecimiento de plantas en el país. 


\section{ReCOMENDACIONES}

Se recomienda para un próximo experimento, evaluar cuántas entradas de vapor son ideales para obtener mejores resultados.

Así mismo, realizar un análisis económico del impacto de la aplicación de vapor en varios puntos para la planta.

Durante el ensayo se utilizaron dietas con alto porcentaje de maíz, casi en un $70 \%$, siendo el maíz un material de fácil acondicionamiento y peletizacion. Se sugiere, entonces, para determinar el comportamiento de la eficiencia del proceso, realizar el ensayo con dietas ricas en otras sustancias de difícil manejo, tales como, lactosa y altos $\%$ de grasas o fibra,.

Se recomienda para analizar la influencia del dado en la calidad de los pellets, es decir, con relación de compresión se puede obtener una mejor calidad de pellets además de las entradas de vapor.

Es importante hacer un estudio de las características reológicas óptimas que debe tener un alimento balanceado, para obtener pellets de buena calidad, teniendo en cuenta los parámetros de elasticidad, textura y consistencia.

Se recomienda el comparativo de esta tecnología con la utilización de acondicionadoras dobles y de expanders.

El tiempo de retención en el acondicionador es una variable muy importante para obtener buenos resultados de calidad y peletizacion de almidones, por lo cual se invita a utilizar tecnología con variación de tiempos de acondicionamiento, y así evaluar en cuál de ellos se observa un mejor resultado en términos de calidad.

\section{ReFERENCIAS}

[1] ICA (2013) ICA. Buenas prácticas en la elaboración de alimentos balanceados. Grupo de regulación y control de alimentos para animales. [Online] Consultado el 15 de Abril de 2013nAvailable: www.ica.gov.co.

[2] Andi, 2012.

[3] P.Joaquín (2013, Noviembre 8) Peletizacion y calidad de pellets, consultado 05 de marzo de 2014. [Online] Available:http://www.engormix.com/MA-balanceados/fabricacion/articulos/peletizacion-calidad-pelett5192/801-p0.htm

[4] B.Eugenio Diseños de plantas de alimentos especializados en peces y crustáceos curso Lance en acuacultura. México, 2001.

[5] S. Jorge. Montaje y puesta en marcha de una planta de alimento balanceado. Universidad católica del Perú. 2008.

[6] K. Behnke. Condition factors affecting pelleting performance and quality pellet. Rapco 2013.

[7] J. Howel y R. Buckius..Principios de Termodinámica para Ingeniería" 1a. Ed. EditorialMcGraw Hill, México (1990).

[8] [O. Giuliani. Entender el proceso de peletizado para lograr mejores resultados. Revista industria avícola. P. 18, Jul. 2011.

[9] R.McEllhiney.. Tecnología para la fabricación de alimentos balanceados.1 ${ }^{\text {a }}$ ed. Kansas StateUniverity, 1994.

[10] ASAE, Wafres, pellets, crumbles, definitions of methods for determination density, durability, and moisture, contend. Asae standar S269.3. American society agricultural engineers. 1987.

[11] (Markus, Rollins 2007).

[12] Fedna.(2013) Tasa de gelatinización de almidones. 1999. [Online] Consultado el 11 de Diciembre de 2013. Availabe: http://www.fundacionfedna.org/tecnicas_de_analisis/tasa-de-gelatinizaci\%C3\%B3n-delalmid\%C3\%B3n 